\title{
Exponential Lower Bounds for Polytopes in Combinatorial Optimization
}

\author{
SAMUEL FIORINI and SERGE MASSAR, Université libre de Bruxelles \\ SEBASTIAN POKUTTA, Georgia Institute of Technology \\ HANS RAJ TIWARY, Université libre de Bruxelles \\ RONALD DE WOLF, CWI and University of Amsterdam
}

\begin{abstract}
We solve a 20 -year old problem posed by Yannakakis and prove that no polynomial-size linear program (LP) exists whose associated polytope projects to the traveling salesman polytope, even if the LP is not required to be symmetric. Moreover, we prove that this holds also for the cut polytope and the stable set polytope. These results were discovered through a new connection that we make between one-way quantum communication protocols and semidefinite programming reformulations of LPs.
\end{abstract}

Categories and Subject Descriptors: F.2.2 [Analysis of Algorithms and Problem Complexity]: Nonnumerical Algorithms and Problems; G.2.0 [Discrete Mathematics]: General

General Terms: Theory

Additional Key Words and Phrases: Combinatorial optimization, linear programming, communication complexity, semidefinite programming, quantum communication complexity

\section{ACM Reference Format:}

Samuel Fiorini, Serge Massar, Sebastian Pokutta, Hans Raj Tiwary, and Ronald de Wolf. 2015. Exponential lower bounds for polytopes in combinatorial optimization. J. ACM 62, 2, Article 17 (April 2015), 23 pages. DOI: http://dx.doi.org/10.1145/2716307

\section{INTRODUCTION}

Since the advent of the simplex method [Dantzig 1951], linear programming has become a prominent tool for solving optimization problems in practice. On the theoretical side, LPs can be solved in polynomial time via either the ellipsoid method [Khachiyan 1979] or interior point methods [Karmarkar 1984].

In 1986-1987, there were attempts [Swart 1987] to prove $\mathrm{P}=\mathrm{NP}$ by giving a polynomial-size LP that would solve the traveling salesman problem (TSP). Due to

The author list is alphabetical.

S. Fiorini acknowledges support from the Actions de Recherche Concertées (ARC) fund of the French community of Belgium. S. Massar acknowledges support from the European Commission under the projects QCS (Grant No. 255961) and QALGO (Grant No. 600700). H. R. Tiwary was a postdoctoral researcher of the Fonds National de la Recherche Scientifique (F.R.S.-FNRS). R. de Wolf was partially supported by a Vidi grant from the Netherlands Organization for Scientific Research (NWO), by ERC Consolidator grant QPROGRESS, and by the European Commission under the projects QCS (Grant No. 255961) and QALGO (Grant No. 600700). Authors' present addresses: S. Fiorini, Université libre de Bruxelles, Département de Mathématique, Algebra and Combinatorics (CP 216), Boulevard du Triomphe, B-1050 Brussels, Belgium; S. Massar, Université libre de Bruxelles, Laboratoire d'information quantique (CP 255), Boulevard du Triomphe, B-1050 Brussels, Belgium; S. Pokutta, Georgia Institute of Technology, Groseclose 0205, Ofice 338, 765 Ferst Drive, Atlanta, GA 30332; H. R. Tiwary, Department of Applied Mathematics, Faculty of Mathematics and Physics, Charles University, Malostranské nám. 25, 11800 Prague 1, Czech Republic; R. de Wolf, CWI, P.O. Box 94079, NL-1090 GB Amsterdam, The Netherlands. Correspondence email: hansrajt@gmail.com.

Permission to make digital or hard copies of part or all of this work for personal or classroom use is granted without fee provided that copies are not made or distributed for profit or commercial advantage and that copies show this notice on the first page or initial screen of a display along with the full citation. Copyrights for components of this work owned by others than ACM must be honored. Abstracting with credit is permitted. To copy otherwise, to republish, to post on servers, to redistribute to lists, or to use any component of this work in other works requires prior specific permission and/or a fee.

2015 Copyright is held by the author/owner(s). Publication rights licensed to ACM.

0004-5411/2015/04-ART17 $\$ 15.00$

DOI: http://dx.doi.org/10.1145/2716307 
the large size and complicated structure of the proposed LP for the TSP, it was difficult to show directly that the LP was erroneous. In a groundbreaking effort to refute all such attempts, Yannakakis [1988] proved that every symmetric LP for the TSP has exponential size (see Yannakakis [1991] for the journal version). Here, an LP is called symmetric if every permutation of the cities can be extended to a permutation of all the variables of the LP that preserves the constraints of the LP. Because the proposed LP for the TSP was symmetric, it could not possibly be correct.

In his paper, Yannakakis left as a main open problem the question of proving that the TSP admits no polynomial-size LP, symmetric or not. We solve this question by proving a super-polynomial lower bound on the number of inequalities in every LP for the TSP. We also prove such unconditional super-polynomial lower bounds for the maximum cut and maximum stable set problems. Therefore, it is impossible to prove $\mathrm{P}=\mathrm{NP}$ by means of a polynomial-size LP that expresses any of these problems. Our approach is inspired by a close connection between semidefinite programming reformulations of LPs and one-way quantum communication protocols that we introduce here.

\subsection{State of the Art}

From Problems to Polytopes. For combinatorial optimization problems such as the TSP, the feasible solutions can be encoded as points in a set $X \subseteq\{0,1\}^{d}$ in such a way that solving an instance of the problem amounts to optimizing a linear objective function over $X$, with coefficients given by the instance. By taking the convex hull of $X$, one obtains a polytope $P:=\operatorname{conv}(X)$ (see Appendix A for background on polytopes). Optimizing any linear function $f(x)$ over $X$ is equivalent to optimizing this function $f(x)$ over $P=\operatorname{conv}(X)$.

For example, for the TSP, we have a set $X \subseteq\{0,1\}^{\left(\begin{array}{c}n \\ 2\end{array}\right)}$ of 0/1-points that correspond to a Hamiltonian cycle in the complete $n$-vertex graph $K_{n}$. The convex hull of these points is the TSP polytope $\operatorname{TSP}(n)=\operatorname{conv}(X)$. An instance of the TSP is given by the set of edge-weights $w_{i j}$. Solving this instance amounts to minimizing $f(x):=\sum_{i<j} w_{i j} x_{i j}$ over all $x \in \operatorname{TSP}(n)$. This minimum is attained at a vertex of the polytope, that is, at a point $x \in X$.

The idea of representing the set of feasible solutions of a problem by a polytope forms the basis of a standard and powerful methodology in combinatorial optimization, see, for example, Schrijver [2003].

Extended Formulations and Extensions. Resuming the previous discussion (and assuming that the problem is a minimization problem), we have $\min \{f(x) \mid x \in X\}=$ $\min \{f(x) \mid x \in P\}=\min \{f(x) \mid A x \leqslant b\}$, where $A x \leqslant b$ is any linear description of $P$. This turns any given instance of the combinatorial optimization problem into an LP, however, over an implicit system of constraints, the LP is potentially large since it has at least one inequality per facet of $P$. In fact, even for polynomially solvable problems, the associated polytope $P$ may have an exponential number of facets.

By working in an extended space, that is, considering extra variables $y \in \mathbb{R}^{k}$ besides the original variables $x \in \mathbb{R}^{d}$, it is often possible to decrease the number of constraints. In some cases, a polynomial increase in dimension can be traded for an exponential decrease in the number of constraints. This is the idea underlying extended formulations.

Formally, an extended formulation (EF) of a polytope $P \subseteq \mathbb{R}^{d}$ is a linear system

$$
E^{=} x+F^{=} y=g^{=}, E^{\leqslant} x+F^{\leqslant} y \leqslant g \leqslant
$$

in variables $(x, y) \in \mathbb{R}^{d+k}$, where $E^{=}, F^{=}, E^{\leqslant}, F^{\leqslant}$are real matrices with $d, k, d, k$ columns respectively, and $g^{=}, g^{\leqslant}$are column vectors, such that $x \in P$ if and only if there exists $y$ such that (1) holds. 
The size of an EF is defined as the number of inequalities in the system. Another possible definition of size would be the sum of the number of variables and total number of constraints (equalities plus inequalities) defining the EF. This would make little difference because if a polytope $P \subseteq \mathbb{R}^{d}$ has an EF with $r$ inequalities, then it has an EF with $d+r$ variables, $r$ inequalities and at most $d+r$ equalities (see Remark 3.1 for a proof). If we assume that $P$ is full-dimensional (otherwise, one may cheat and make $d$ artificially high), then $d \leqslant r$ and thus the two measures of size are within a constant of each other.

Notice that optimizing any (not necessarily linear) objective function $f(x)$ over all $x \in P$ amounts to optimizing $f(x)$ over all $(x, y) \in \mathbb{R}^{d+k}$ satisfying (1), provided (1) defines an EF of $P$.

Here, we often restrict to EFs in slack form, that is, containing only equalities and one nonnegativity inequality per additional variable:

$$
E x+F y=g, y \geqslant \mathbf{0} .
$$

The proof of the factorization theorem (Theorem 3 ) shows that this can be done without loss of generality, see Remark 3.1. In the following, we put EFs in slack form to ease the generalization to arbitrary cones. Notice that the size of an EF in slack form can equivalently be defined as the number of additional variables since the only inequalities are from $y \geqslant \mathbf{0}$.

An extension of the polytope $P$ is another polytope $Q \subseteq \mathbb{R}^{e}$ such that $P$ is the image of $Q$ under a linear map. We define the size of an extension $Q$ as the number of facets of $Q$. If $P$ has an extension of size $r$, then it has an EF of size $r$. Conversely, it is known that if $P$ has an EF of size $r$, then it has an extension of size at most $r$ (see Theorem 3 ). In this sense, the concepts of $\mathrm{EF}$ and extension are equivalent.

The Impact of Extended Formulations. EFs have pervaded the areas of discrete optimization and approximation algorithms for a long time. For instance, Balas' disjunctive programming [Balas 1985], the Sherali-Adams hierarchy [Sherali and Adams 1990], the Lovász-Schrijver closures [Lovász and Schrijver 1991], lift-and-project [Balas et al. 1993], and configuration LPs are all based on the idea of working in an extended space. Recent surveys on EFs in the context of combinatorial optimization and integer programming are Conforti et al. [2010], Vanderbeck and Wolsey [2010], Kaibel [2011], and Wolsey [2011].

Symmetry Matters. Yannakakis [1991] proved a $2^{\Omega(n)}$ lower bound on the size of any symmetric EF of the TSP polytope TSP $(n)$ (defined previously and in Section 3.4). Although he remarked that he did "not think that asymmetry helps much", it was recently shown by Kaibel et al. [2010] (see also Pashkovich [2009]) that symmetry is a restriction in the sense that there exist polytopes that have polynomial-size EFs but no polynomial-size symmetric EF. This revived Yannakakis's tantalizing question about unconditional lower bounds. That is, bounds which apply to the extension complexity of a polytope $P$, defined as the minimum size of an EF of $P$ (irrespective of any symmetry assumption).

0/1-Polytopes with Large Extension Complexity. The strongest unconditional lower bounds so far were obtained by Rothvoss [2011]. By an elegant counting argument inspired by Shannon's theorem [Shannon 1949], it was proved that there exist 0/1polytopes in $\mathbb{R}^{d}$ whose extension complexity is at least $2^{d / 2-o(d)}$. However, Rothvoß's counting technique does not provide explicit 0/1-polytopes with an exponential extension complexity.

The Factorization Theorem. Yannakakis [1991] discovered that the extension complexity of a polytope $P$ is determined by certain factorizations of an associated matrix, 
called the slack matrix of $P$, that records for each pair $(F, v)$ of a facet $F$ and vertex $v$, the algebraic distance of $v$ to a valid hyperplane supporting $F$. Defining the nonnegative rank of a matrix $M$ as the smallest natural number $r$ such that $M$ can be expressed as $M=T U$ where $T$ and $U$ are nonnegative matrices (i.e., matrices whose elements are all nonnegative) with $r$ columns (in case of $T$ ) and $r$ rows (in case of $U$ ), respectively, it turns out that the extension complexity of every polytope $P$ is exactly the nonnegative rank of its slack matrix.

We point out that this result generalizes to any slack matrix of the polytope, which may contain additional rows corresponding to faces $F$ of $P$ which are not facets and/or additional columns corresponding to points $v$ of $P$ that are not vertices. This fact is used in the proof of our lower bounds on extension complexity, starting with Theorem 7.

This factorization theorem led Yannakakis to explore connections between EFs and communication complexity. Let $S$ denote the slack matrix of the polytope $P$. He proved that: (i) every deterministic communication protocol of complexity $k$ computing $S$ gives rise to an $\mathrm{EF}$ of $P$ of size at most $2^{k}$; (ii) the nondeterministic communication complexity of the support matrix of $S$ (i.e., the binary matrix that has 0 -entries exactly where $S$ is 0 ) yields a lower bound on (the base-2 logarithm ${ }^{1}$ of) the extension complexity of $P$, or more generally, the nondeterministic communication complexity of the support matrix of every nonnegative matrix $M$ yields a lower bound on (the base-2 logarithm of) the nonnegative rank of $M .^{2}$

Tighter Communication Complexity Connection. Faenza et al. [2011] proved that the base-2 logarithm of the nonnegative rank of a matrix equals, up to a small additive constant, the minimum complexity of a randomized communication protocol with nonnegative outputs that computes the matrix in expectation. In particular, every EF of size $r$ can be regarded as such a protocol of complexity $\log r+O(1)$ bits that computes a slack matrix in expectation.

The Clique vs. Stable Set Problem. When $P$ is the stable set polytope $\operatorname{STAB}(G)$ of a graph $G$ (see Section 3.3), the slack matrix of $P$ contains an interesting row-induced 0/1-submatrix that is the communication matrix of the clique vs. stable set problem (also known as the clique vs. independent set problem): its rows correspond to the cliques and its columns to the stable sets (or independent sets) of graph $G$. The entry for a clique $K$ and stable set $S$ equals $1-|K \cap S|$. Yannakakis [1991] gave an $O\left(\log ^{2} n\right)$ deterministic protocol for the clique vs. stable set problem, where $n$ denotes the number of vertices of $G$. This gives a $2^{O\left(\log ^{2} n\right)}=n^{O(\log n)}$ size $\operatorname{EF}$ for $\operatorname{STAB}(G)$ whenever the whole slack matrix is $0 / 1$, that is, whenever $G$ is a perfect graph.

A notoriously hard open question is to determine the communication complexity (in the deterministic or nondeterministic sense) of the clique vs. stable set problem. (For recent results that explain why this question is hard, see Kushilevitz and Weinreb [2009a, 2009b].) The best lower bound to this day is due to Huang and Sudakov [2012]: they obtained a $\frac{6}{5} \log n-O(1)$ lower bound. Furthermore, they state a graph-theoretical conjecture that, if true, would imply a $\Omega\left(\log ^{2} n\right)$ lower bound, and hence settle the communication complexity of the clique vs. stable set problem. Moreover it would give a worst-case $n^{\Omega(\log n)}$ lower bound on the extension complexity of stable set polytopes. However, a solution to the Huang-Sudakov conjecture seems far away.

\footnotetext{
${ }^{1}$ All logarithms in this article are in base 2.

${ }^{2}$ The classical nondeterministic communication complexity of a binary communication matrix is defined as $\lceil\log B\rceil$, where $B$ is the minimum number of monochromatic 1-rectangles that cover the matrix, see Kushilevitz and Nisan [1997]. This last quantity is also known as the rectangle covering bound. It is easy to see that the rectangle covering bound of the support matrix of any matrix $M$ lower bounds the nonnegative rank of $M$ (see Theorem 4 ).
} 
Factorization Theorem for General Cones. Gouveia et al. [2013] generalized Yannakakis's factorization theorem to other convex cones. There, the question is to know which polytopes $P \subseteq \mathbb{R}^{d}$ can be described via a conic extended formulation

$$
E x+F y=g, y \in C
$$

for some given closed, convex cone $C \subseteq \mathbb{R}^{k}$. Cone $C$ is said to be nice if $C^{*}+F^{\perp}$ is closed for all faces $F$ of $C$, where $C^{*}$ is the $\bar{d}$ ual cone of $C$. It is known that the nonnegative orthants and the PSD cones are nice. Gouveia et al. [2013] prove that, in case $C$ is nice and $P$ has dimension at least 1 , such a conic EF exists if and only if the slack matrix $S$ of $P$ admits a factorization $S=T U$ where (the transpose of) each row of $T$ is in $C^{*}$ and each column of $U$ is in $C$. This implies the following factorization theorem for semidefinite EFs: the semidefinite extension complexity of every polytope $P$ equals the $P S D$ rank of its slack matrix $S$ (see Theorem 13).

\subsection{Our Contribution}

Our contribution in this article is twofold.

-First, inspired by earlier work [de Wolf 2003], we define a $2^{n} \times 2^{n}$ matrix $M=M(n)$ and show that the nonnegative rank of $M$ is $2^{\Omega(n)}$ because the nondeterministic communication complexity of its support matrix is $\Omega(n)$. The latter was proved by de Wolf [2003] using the well-known disjointness lower bound of Razborov [1992]. We use the matrix $M$ to prove a $2^{\Omega(n)}$ lower bound on the extension complexity of the cut polytope $\operatorname{CUT}(n)$ (Section 3.2). That is, we prove that every EF of the cut polytope has an exponential number of inequalities. Via reductions, we infer from this: (i) an infinite family of graphs $G$ such that the extension complexity of the corresponding stable set polytope $\operatorname{STAB}(G)$ is $2^{\Omega(\sqrt{n})}$, where $n$ denotes the number of vertices of $G$ (Section 3.3); (ii) that the extension complexity of the TSP polytope $\operatorname{TSP}(n)$ is $2^{\Omega(\sqrt{n})}$ (Section 3.4).

In addition to simultaneously settling the previously mentioned open problems of Yannakakis [1991] and Rothvoss [2011], our results provide a lower bound on the extension complexity of stable set polytopes that goes much beyond what is implied by the Huang-Sudakov conjecture (thanks to the fact that we consider a different part of the slack matrix). Although our lower bounds are strong, unconditional, and apply to explicit polytopes that are well known in combinatorial optimization, they have very accessible proofs.

- Second, we generalize the tight connection between linear ${ }^{3}$ EFs and classical communication complexity found by Faenza et al. [2011] to a tight connection between semidefinite EFs and quantum communication complexity. ${ }^{4}$ We show that any rank$r$ PSD factorization of a (nonnegative) matrix $M$ gives rise to a one-way quantum protocol computing $M$ in expectation that uses $\log r+O(1)$ qubits and, conversely, that any one-way quantum protocol computing $M$ in expectation that uses $q$ qubits results in a PSD factorization of $M$ of rank $2^{q}$. Via the semidefinite factorization theorem, this yields a characterization of the semidefinite extension complexity of a polytope in terms of the minimum complexity of (one-way) quantum protocols that compute the corresponding slack matrix in expectation.

\footnotetext{
${ }^{3}$ In this paragraph, and later in Section 4, an EF (in the sense of the previous section) is called a linear EF. The use of adjectives such as "linear," "semidefinite," or "conic" will help distinguishing the different types of EFs.

${ }^{4}$ After a first version of this article appeared, Jain et al. [2013, Theorem 2] have used this notion of PSD rank to characterize the number of qubits of communication between Alice and Bob needed to generate a shared probability distribution.
} 
Then, we give a complexity $\log r+O(1)$ quantum protocol for computing a nonnegative matrix $M$ in expectation, whenever there exists a rank- $r$ matrix $N$ such that $M$ is the entry-wise square of $N$. This implies in particular that every $d$-dimensional polytope with $0 / 1$ slacks has a semidefinite EF of size $O(d)$.

Finally, we obtain an exponential separation between classical and quantum protocols that compute our specific matrix $M=M(n)$ in expectation. On the one hand, our quantum protocol gives a rank- $O(n)$ PSD factorization of $M$. On the other hand, the nonnegative rank of $M$ is $2^{\Omega(n)}$ because the nondeterministic communication complexity of the support matrix of $M$ is $\Omega(n)$. Thus, we also obtain an exponential separation between PSD rank and nonnegative rank.

We would like to point out that the lower bounds on the extension complexity of polytopes established in Section 3 were obtained by first finding an efficient PSD factorization or, equivalently, an efficient one-way quantum communication protocol for the matrix $M=M(n)$. In this sense, our classical lower bounds stem from quantum considerations somewhat similar in style to Kerenidis and de Wolf [2004], Aaronson [2006], and Aharonov and Regev [2004]. See Drucker and de Wolf [2011] for a survey of this line of work.

We would also like to point out that the fact that a matrix $M$ with a rank- $r$ entrywise square-root has a PSD-rank at most $r+O(1)$, which follows from Theorem 16, was also obtained by Gouveia et al. [2013], independently (since their results were not publicly available at the time we performed our research) and in a different context. Also, after a preprint of our paper had appeared, we learned that Klauck et al. [2011] had independently found a matrix (similar but not quite the same as ours) with an exponential separation between PSD rank and nonnegative rank.

\subsection{Other Related and Subsequent Work}

Yannakakis's paper has deeply influenced the TCS community. In addition to the works cited previously, it has inspired a whole series of papers on the quality of restricted approximate EFs, such as those defined by the Sherali-Adams hierarchies and LovászSchrijver closures starting with Arora et al. [2002] (Arora et al. [2006] for the journal version), see, for example, Buresh-Oppenheim et al. [2006], Schoenebeck et al. [2007], Fernandez de la Vega and Mathieu [2007], Charikar et al. [2009], Georgiou et al. [2009, 2010], and Benabbas and Magen [2010].

After the conference version of our article appeared, there has been a lot of follow-up work, including on approximations. Braun et al. [2012] developed a general framework for studying the power of approximate EFs, independent of specific hierarchies. In particular, via lower bounds on the extension complexity of approximations of the cut polytope, they showed that linear programs for approximating Max-Clique to within a factor $n^{1 / 2-\epsilon}$ need size at least $2^{\Omega\left(n^{\epsilon}\right)}$. Similarly, they show the existence of a spectrahedron of small size that cannot be approximated by any LP with a polynomial number of inequalities within a factor of $n^{1 / 2-\epsilon}$. Braverman and Moitra [2013] used methods from information complexity to show the same size lower bound even for approximation factor $n^{1-\epsilon}$; Braun and Pokutta [2013] subsequently simplified and generalized their result and Braun et al. [2013b] show that the amortized log nonnegative rank is characterized by information. Such inapproximability results should be contrasted with Håstad's famous result [Håstad 1999] that it is hard to approximate Max-Clique to within a factor $n^{1-\epsilon}$ : Håstad's result gives is a lower bound for all algorithms approximating Max-Clique and is conditional on the unproven assumption that $\mathrm{RP} \neq \mathrm{NP}$, while the results of Braun et al. [2012] and Braverman and Moitra [2013] are geometric statements about the nonexistence of polynomial-size extended formulations. 
Braun et al. [2013a] analyze the average-case polyhedral complexity of the maximum stable set problem showing that the extension complexity of the stable set polytope is high for almost all graphs. Pokutta and Van Vyve [2013] proved lower bounds on extension complexity for the knapsack problem, and Avis and Tiwary [2013] proved lower bounds for the subset-sum and three-dimensional matching problems, as well as others. Kaibel and Weltge [2013] gave a more direct proof of the lower bound for the cut polytope, via bounding the measure of the largest rectangle in the slack matrix, under the condition that this rectangle is contained in the support. However, they still use the same set of $2^{n}$ valid constraints that we use here (Lemma 6).

Chan et al. [2013] prove super-polynomial lower bounds on approximate EFs for MAX CSPs (constraint satisfaction problems). In particular, they prove that every $(2-\varepsilon)$-approximate (linear) EF for Max-Cut has $n^{\Omega\left(\frac{\log n}{\log \log n}\right)}$ size. This is striking because the celebrated approximation algorithm of Goemans and Williamson [1995] is based on a $\Theta(n)$-size semidefinite EF with an approximation factor of at most 1.14. Again, the result of Chan et al. [2013] on Max-Cut matches the algorithmic hardness of the problem Khot et al. [2007], which assumes the Unique Games Conjecture.

Rothvoss [2014] proves that the matching polytope has extension complexity $2^{\Omega(n)}$, solving the second part of the main open problem in Yannakakis [1991]. This is the first time such a strong bound is obtained for a polytope over which one can optimize in polynomial time. Rothvoß's groundbreaking result implies in particular that the extension complexity of the TSP polytope is $2^{\Omega(n)}$, thus going beyond our $2^{\Omega(\sqrt{n})}$ lower bound.

Not much is known yet about lower bounds on semidefinite EFs. Extending the work of Rothvoss [2011] and Briët et al. [2013] show that most 0/1 polytopes (i.e., polytopes that are the convex hull of a random subset of $\{0,1\}^{d}$ ) need exponentially large semidefinite EFs. Fawzi and Parrilo [2013] give exponential lower bounds on the size of semidefinite EFs of explicit polytopes in a restricted setting, where the underlying cone is not the full PSD cone but rather a product of fixed-dimensional PSD cones. Lee and Theis [2012] obtain polynomial lower bounds based on the support pattern of slack matrices.

Finally, Fiorini et al. [2013] use the notion of conic extensions and its relation to communication complexity to study generalized probabilistic theories, which are different from the usual classical or quantum-mechanical theories, and show that all polynomially-definable 0/1-polytopes have small extension complexity with respect to the completely positive cone.

\subsection{Organization}

The discovery of our lower bounds on extension complexity crucially relied on finding the right matrix $M$ and the right polytope whose slack matrix contains $M$. In our case, we found these through a connection with quantum communication. However, these quantum aspects are not strictly necessary for the resulting lower bound proof itself. Hence, in order to make the main results more accessible to those without background or interest in quantum computing, we start by giving a purely classical presentation of those lower bounds.

In Section 2, we define our matrix $M$ and lower bound the nondeterministic communication complexity of its support matrix. In Section 3 , we embed $M$ in the slack matrix of the cut polytope in order to lower bound its extension complexity; further reductions then give lower bounds on the extension complexities of the stable set, and TSP polytopes. In Section 4, we establish the equivalence of PSD factorizations of a (nonnegative) matrix $M$ and one-way quantum protocols that compute $M$ in expectation, and give an efficient quantum protocol in the case where 
some entry-wise square root of $M$ has small rank. This is then used to provide an exponential separation between quantum and classical protocols for computing a matrix in expectation (equivalently, an exponential separation between nonnegative rank and PSD rank). Concluding remarks are given in Section 5.

\section{A SIMPLE MATRIX WITH LARGE RECTANGLE COVERING BOUND}

In this section we consider the following $2^{n} \times 2^{n}$ matrix $M=M(n)$ with rows and columns indexed by $n$-bit strings $a$ and $b$, and real nonnegative entries:

$$
M_{a b}:=\left(1-a^{\top} b\right)^{2} .
$$

Note for later reference that $M_{a b}$ can also be written as

$$
M_{a b}=1-\left\langle 2 \operatorname{diag}(a)-a a^{\top}, b b^{\top}\right\rangle,
$$

where $\langle\cdot, \cdot\rangle$ denotes the Frobenius inner $\operatorname{product}^{5}$ and $\operatorname{diag}(a)$ is the $n \times n$ diagonal matrix with the entries of $a$ on its diagonal. Let us verify this identity, using $a, b \in\{0,1\}^{n}$ :

$$
\begin{aligned}
1- & \left\langle 2 \operatorname{diag}(a)-a a^{\top}, b b^{\top}\right\rangle \\
& =1-2\left\langle\operatorname{diag}(a), b b^{\top}\right\rangle+\left\langle a a^{\top}, b b^{\top}\right\rangle \\
& =1-2 a^{\top} b+\left(a^{\top} b\right)^{2}=\left(1-a^{\top} b\right)^{2} .
\end{aligned}
$$

Let $\operatorname{suppmat}(M)$ be the binary support matrix of $M$, so

$$
\operatorname{suppmat}(M)_{a b}=\left\{\begin{array}{l}
1 \text { if } M_{a b} \neq 0, \\
0 \text { otherwise. }
\end{array}\right.
$$

For a given matrix, a rectangle is the Cartesian product of a set of row indices and a set of column indices. In de Wolf [2003] it was shown that an exponential number of (monochromatic) rectangles are needed to cover all the 1-entries of the support matrix of $M$. Equivalently, the corresponding function $f:\{0,1\}^{n} \times\{0,1\}^{n} \rightarrow\{0,1\}$ has nondeterministic communication complexity of $\Omega(n)$ bits. For the sake of completeness, we repeat the proof here.

THeOREM 1 [DE Wolf 2003]. Every 1-monochromatic rectangle cover of $\operatorname{suppmat}(M)$ has size $2^{\Omega(n)}$.

Proof. Let $R_{1}, \ldots, R_{k}$ be a 1-cover for $f$, that is, a set of (possibly overlapping) 1monochromatic rectangles in the matrix $\operatorname{suppmat}(M)$ that together cover all 1-entries in suppmat $(M)$.

We use the following result from Kushilevitz and Nisan [1997, Example 3.22 and Section 4.6], which is essentially due to Razborov [1992].

There exist sets $A, B \subseteq\{0,1\}^{n} \times\{0,1\}^{n}$ and probability distribution $\mu$ on $\{0,1\}^{n} \times\{0,1\}^{n}$ such that all $(a, b) \in A$ have $a^{\top} b=0$, all $(a, b) \in B$ have $a^{\top} b=1, \mu(A)=3 / 4$, and there are constants $\alpha, \delta>0$ (independent of $n$ ) such that for all rectangles $R$,

$$
\mu(R \cap B) \geqslant \alpha \cdot \mu(R \cap A)-2^{-\delta n} .
$$

(For sufficiently large $n, \alpha=1 / 135$ and $\delta=0.017$ will do.)

\footnotetext{
${ }^{5}$ The Frobenius inner product is the component-wise inner product of two matrices. For matrices $X$ and $Y$ of the same dimensions, this equals $\operatorname{Tr}\left[X^{\top} Y\right]$. When $X$ is symmetric, this can also be written $\operatorname{Tr}[X Y]$.
} 
Since the $R_{i}$ are 1-rectangles, they cannot contain elements from $B$. Hence, $\mu\left(R_{i} \cap B\right)=0$ and $\mu\left(R_{i} \cap A\right) \leqslant 2^{-\delta n} / \alpha$. However, since all elements of $A$ are covered by the $R_{i}$, we have

$$
\frac{3}{4}=\mu(A)=\mu\left(\bigcup_{i=1}^{k}\left(R_{i} \cap A\right)\right) \leqslant \sum_{i=1}^{k} \mu\left(R_{i} \cap A\right) \leqslant k \cdot \frac{2^{-\delta n}}{\alpha} .
$$

Hence, $k \geqslant 2^{\Omega(n)}$.

\section{STRONG LOWER BOUNDS ON EXTENSION COMPLEXITY}

Here we use the matrix $M=M(n)$ defined in the previous section to prove that the (linear) extension complexity of the cut polytope of the $n$-vertex complete graph is $2^{\Omega(n)}$, that is, every (linear) EF of this polytope has an exponential number of inequalities. Then, via reductions, we prove super-polynomial lower bounds for the stable set polytopes and the TSP polytopes. To start, let us define more precisely the slack matrix of a polytope. For a matrix $A$, let $A_{i}$ denote the $i$ th row of $A$ and let $A^{j}$ denote the $j$ th column of $A$.

Let $P=\left\{x \in \mathbb{R}^{d} \mid A x \leqslant b\right\}=\operatorname{conv}(V)$ be a polytope, with $A \in \mathbb{R}^{m \times d}, b \in \mathbb{R}^{m}$ and $V=$ $\left\{v_{1}, \ldots, v_{n}\right\} \subseteq \mathbb{R}^{d}$. Then $S \in \mathbb{R}_{+}^{m \times n}$ defined as $S_{i j}:=b_{i}-A_{i} v_{j}$ with $i \in[m]:=\{1, \ldots, m\}$ and $j \in[n]:=\{1, \ldots, n\}$ is the slack matrix of $P$ with respect to $A x \leqslant b$ and $V$. We sometimes refer to the submatrix of the slack matrix induced by rows corresponding to facets and columns corresponding to vertices simply as the slack matrix of $P$, denoted by $S(P)$.

Recall that

(1) an extended formulation (EF) of $P$ is a linear system in variables $(x, y)$ such that $x \in P$ if and only if there exists $y$ satisfying the system;

(2) an extension of $P$ is a polytope $Q \subseteq \mathbb{R}^{e}$ such that there is a linear map $\pi: \mathbb{R}^{e} \rightarrow \mathbb{R}^{d}$ with $\pi(Q)=P$

(3) the extension complexity of $P$ is the minimum size (i.e., number of inequalities) of an EF of $P$.

We denote the extension complexity of $P$ by $x c(P)$.

\subsection{The Factorization Theorem}

A rank-r nonnegative factorization of a (nonnegative) matrix $M$ is a factorization $M=$ $T U$ where $T$ and $U$ are nonnegative matrices with $r$ columns (in case of $T$ ) and $r$ rows (in case of $U$ ), respectively. The nonnegative rank of $M$, denoted by $\operatorname{rank}_{+}(M)$, is thus simply the minimum rank among all nonnegative factorizations of $M$. Note that $\operatorname{rank}_{+}(M)$ is also the minimum $r$ such that $M$ is the sum of $r$ nonnegative rank-1 matrices. In particular, the nonnegative rank of a matrix $M$ is at least the nonnegative rank of any submatrix of $M$.

The following factorization theorem was proved by Yannakakis (see also Fiorini et al. [2011]). It can be stated succinctly as: $\mathrm{xc}(P)=\operatorname{rank}_{+}(S)$ whenever $P$ is a polytope and $S$ a slack matrix of $P$. We include a sketch of the proof for completeness and we will use the following lemma which follows easily from Farkas's Lemma [Schrijver 2003; Ziegler 1995] by first showing that $\mathbf{0}^{\top} x \leqslant 1$ can be derived from the system.

Lemma 2. Let $P=\left\{x \in \mathbb{R}^{d} \mid A x \leqslant b\right\}$ be a (possibly unbounded) polyhedron that admits a direction $u \in \mathbb{R}^{d}$ with $-\infty<\min \left\{u^{\top} x \mid x \in P\right\}<\max \left\{u^{\top} x \mid x \in P\right\}<+\infty$, and $c^{\top} x \leqslant \delta$ a valid inequality for $P$. Then there exist nonnegative multipliers $\lambda \in \mathbb{R}^{d}$ such that $\lambda^{\top} A=c^{\top}$ and $\lambda^{\top} b=\delta$, that is, $c^{\top} x \leqslant \delta$ can be derived as a nonnegative combination from $A x \leqslant b$. In particular, this holds whenever $P$ is a polytope of dimension at least 1 or 
whenever $P$ is an unbounded polyhedron that linearly projects to a polytope of dimension at least 1.

We are ready to state Yannakakis's factorization theorem.

Theorem 3 [YANnAKakis 1991]. Let $P=\left\{x \in \mathbb{R}^{d} \mid A x \leqslant b\right\}=\operatorname{conv}(V)$ be a polytope with $\operatorname{dim}(P) \geqslant 1$, and let $S$ denote the slack matrix of $P$ with respect to $A x \leqslant b$ and $V$. Then the following are equivalent for all positive integers $r$ :

(i) $S$ has nonnegative rank at most $r$;

(ii) $P$ has an extension of size at most $r$ (i.e., with at most $r$ facets);

(iii) $P$ has an EF of size at most $r$ (i.e., with at most $r$ inequalities).

Proof. It should be clear that (ii) implies (iii). We prove that (i) implies (ii), and then that (iii) implies (i).

First, consider a rank- $r^{*}$ nonnegative factorization $S=T U$ of the slack matrix of $P$, where $r^{*} \leqslant r$. Notice that we may assume that no column of $T$ is zero, because otherwise $r^{*}$ can be decreased. We claim that $P$ is the image of

$$
Q:=\left\{(x, y) \in \mathbb{R}^{d+r^{*}} \mid A x+T y=b, y \geqslant \mathbf{0}\right\}
$$

under the projection $\pi_{x}:(x, y) \mapsto x$ onto the $x$-space. We see immediately that $\pi_{x}(Q) \subseteq P$ since $T y \geqslant \mathbf{0}$. To prove the inclusion $P \subseteq \pi_{x}(Q)$, it suffices to remark that for each point $v_{j} \in V$ the point $\left(v_{j}, U^{j}\right)$ is in $Q$ since

$$
A v_{j}+T U^{j}=A v_{j}+b-A v_{j}=b \text { and } U^{j} \geqslant \mathbf{0} .
$$

Since no column of $T$ is zero, $Q$ is a polytope. Moreover, $Q$ has at most $r^{*} \leqslant r$ facets, and is thus an extension of $P$ of size at most $r$. This proves that (i) implies (ii).

Second, suppose that the system

$$
E^{=} x+F^{=} y=g^{=}, E^{\leqslant} x+F^{\leqslant} y \leqslant g \leqslant
$$

with $(x, y) \in \mathbb{R}^{d+k}$ defines an EF of $P$ with at most $r$ inequalities. Let $Q \subseteq \mathbb{R}^{d+k}$ denote the set of solutions to this system. Thus, $Q$ is a (not necessarily bounded) polyhedron. For each point $v_{j} \in V$, pick $w_{j} \in \mathbb{R}^{k}$ such that $\left(v_{j}, w_{j}\right) \in Q$. Because

$$
A x \leqslant b \Longleftrightarrow \exists y: E^{=} x+F^{=} y=g^{=}, E^{\leqslant} x+F^{\leqslant} y \leqslant g \leqslant,
$$

each inequality in $A x \leqslant b$ is valid for all points of $Q$. Let $S_{Q}$ be the nonnegative matrix that records the slacks of the points $\left(v_{j}, w_{j}\right)$ with respect to the inequalities of $E \leqslant x+F \leqslant y \leqslant g \leqslant$, and then of $A x \leqslant b$. By construction, the submatrix obtained from $S_{Q}$ by deleting the rows corresponding to the inequalities of $E \leqslant x+F \leqslant y \leqslant g \leqslant$ and leaving only those corresponding to the inequalities of $A x \leqslant b$ is exactly $S$, thus $\operatorname{rank}_{+}(S) \leqslant \operatorname{rank}_{+}\left(S_{Q}\right)$. Furthermore, by Lemma 2 , any valid inequality $c^{\top} x \leqslant \delta$ is a nonnegative combination of inequalities of the system $A x \leqslant b$ and thus every row of $S_{Q}$ is a nonnegative combination of the first $r$ rows of $S_{Q}$. Thus, $\operatorname{rank}_{+}\left(S_{Q}\right) \leqslant r$. Therefore, $\operatorname{rank}_{+}(S) \leqslant r$. Hence, (iii) implies (i).

Remark 3.1. By the factorization theorem, if polytope $P \subseteq \mathbb{R}^{d}$ has an EF of size $r$, then its slack matrix $S$ has a nonnegative factorization $S=T U$ of rank $r$. But then $A x+T y=b, y \geqslant \mathbf{0}$ is an EF of $P$ in slack form with $d+r$ variables, $r$ inequalities and $m$ equalities, where $m$ is the number of rows in the linear description $A x \leqslant b$ of $P$. Notice that if $m>d+r$ some of these equalities will be redundant, and that there always exists a subset of at most $d+r$ equalities defining the same subspace. By removing redundant equalities from the $\mathrm{EF}$, we can assume that there are at most $d+r$ equalities in the EF. 
We would like to emphasize that we will not restrict the slack matrix to have rows corresponding only to the facet-defining inequalities. This is not an issue since appending rows corresponding to redundant ${ }^{6}$ inequalities does not change the nonnegative rank of the slack matrix. This fact was already used in the second part of the previous proof.

Theorem 3 shows in particular that we can lower bound the extension complexity of $P$ by lower bounding the nonnegative rank of its slack matrix $S$; in fact, it suffices to lower bound the nonnegative rank of any submatrix of the slack matrix $S$ corresponding to an implied system of inequalities. To that end, Yannakakis made the following connection with nondeterministic communication complexity. Again, we include the (easy) proof for completeness.

TheORem 4 [YANNAKAKIS 1991]. Let $M$ be any matrix with nonnegative real entries and $\operatorname{suppmat}(M)$ its support matrix. Then $\operatorname{rank}_{+}(M)$ is lower bounded by the rectangle covering bound for suppmat $(M)$.

PRoof. If $M=T U$ is a rank-r nonnegative factorization of $M$, then $S$ can be written as the sum of $r$ nonnegative rank-1 matrices:

$$
S=\sum_{k=1}^{r} T^{k} U_{k}
$$

Taking the support on each side, we find

$$
\begin{aligned}
\operatorname{supp}(S) & =\bigcup_{k=1}^{r} \operatorname{supp}\left(T^{k} U_{k}\right) \\
& =\bigcup_{k=1}^{r} \operatorname{supp}\left(T^{k}\right) \times \operatorname{supp}\left(U_{k}\right)
\end{aligned}
$$

Therefore, $\operatorname{suppmat}(M)$ has a 1-monochromatic rectangle cover with $r$ rectangles.

\subsection{Cut and Correlation Polytopes}

Let $K_{n}=\left(V_{n}, E_{n}\right)$ denote the $n$-vertex complete graph. For a set $X$ of vertices of $K_{n}$, we let $\delta(X)$ denote the set of edges of $K_{n}$ with one endpoint in $X$ and the other in its complement $\bar{X}$. This set $\delta(X)$ is known as the cut defined by $X$. For a subset $F$ of edges of $K_{n}$, we let $\chi^{F} \in \mathbb{R}^{E_{n}}$ denote the characteristic vector of $F$, with $\chi_{e}^{F}=1$ if $e \in F$ and $\chi_{e}^{F}=0$ otherwise. The cut polytope $\operatorname{CUT}(n)$ is defined as the convex hull of the characteristic vectors of all cuts in the complete graph $K_{n}=\left(V_{n}, E_{n}\right)$. That is,

$$
\operatorname{CUT}(n):=\operatorname{conv}\left\{\chi^{\delta(X)} \in \mathbb{R}^{E_{n}} \mid X \subseteq V_{n}\right\} .
$$

We will not deal with the cut polytopes directly, but rather with 0/1-polytopes that are linearly isomorphic to them. Two polytopes are called linearly isomorphic if one can be obtained from the other by applying an invertible linear map. It is easy to check that, if $P_{1}$ and $P_{2}$ are linearly isomorphic, then they have same number of vertices and facets. Furthermore, any extended formulation for one can be converted to an extended formulation of the other using the same transformation. So any bound on the extension complexity of one polytope applies to any other polytope that is linearly isomorphic to it. The correlation polytope (or Boolean quadric polytope) $\mathrm{COR}(n)$ is defined as the convex

\footnotetext{
${ }^{6} \mathrm{An}$ inequality of a linear system is called redundant if removing the inequality from the system does not change the set of solutions.
} 
hull of all the rank-1 binary symmetric matrices of size $n \times n$. In other words,

$$
\operatorname{COR}(n):=\operatorname{conv}\left\{b b^{\top} \in \mathbb{R}^{n \times n} \mid b \in\{0,1\}^{n}\right\} .
$$

We use the following known result.

Theorem 5 [De Simone 1990]. For all n, COR $(n)$ is linearly isomorphic to CUT $(n+1)$.

Consider the matrix $M$ defined in Section 2. Because $M$ is nonnegative, Eq. (4) gives us a linear inequality that is satisfied by all vertices $b b^{\top}$ of $\operatorname{COR}(n)$, and hence (by convexity) is satisfied by all points of $\operatorname{COR}(n)$ :

Lemma 6. For all $a \in\{0,1\}^{n}$, the inequality

$$
\left\langle 2 \operatorname{diag}(a)-a a^{\top}, x\right\rangle \leqslant 1
$$

is valid for $\operatorname{COR}(n)$. Moreover, the slack of vertex $x=b b^{\top}$ with respect to (5) is precisely $M_{a b}$.

We remark that (5) is weaker than the hypermetric inequality [Deza and Laurent 1997] $\left\langle\operatorname{diag}(a)-a a^{\top}, x\right\rangle \leqslant 0$, in the sense that the face defined by the former is strictly contained in the face defined by the latter. Nevertheless, we persist in using (5). Now, we prove the main result of this section.

THEOREM 7. There exists some constant $C>0$ such that, for all $n$,

$$
\operatorname{xc}(\operatorname{CUT}(n+1))=\operatorname{xc}(\operatorname{COR}(n)) \geqslant 2^{C n} .
$$

In particular, the extension complexity of $\mathrm{CUT}(n)$ is $2^{\Omega(n)}$.

Proof. The equality is implied by Theorem 5. Now, consider any system of linear inequalities describing $\operatorname{COR}(n)$ starting with the $2^{n}$ inequalities (5), and a slack matrix $S$ with respect to this system and $\left\{b b^{\top} \mid b \in\{0,1\}^{n}\right\}$. Next delete from this slack matrix all rows except the $2^{n}$ first rows. By Lemma 6 , the resulting $2^{n} \times 2^{n}$ matrix is $M$. Using Theorems 3, 4, and 1, and the fact that the nonnegative rank of a matrix is at least the nonnegative rank of any of its submatrices, we have

$$
\begin{aligned}
\operatorname{xc}(\operatorname{COR}(n)) & =\operatorname{rank}_{+}(S) \\
& \geqslant \operatorname{rank}_{+}(M) \\
& \geqslant 2^{C n}
\end{aligned}
$$

for some positive constant $C$.

In their follow-up work, Kaibel and Weltge [2013] proved that one can take $C=$ $\log (3 / 2) \approx 0.58$.

\subsection{Stable Set Polytopes}

A stable set $S$ (also called an independent set) of a graph $G=(V, E)$ is a subset $S \subseteq V$ of the vertices such that no two of them are adjacent. For a subset $S \subseteq V$, we let $\chi^{\bar{S}} \in \mathbb{R}^{V}$ denote the characteristic vector of $S$, with $\chi_{v}^{S}=1$ if $v \in S$ and $\chi_{v}^{S}=0$ otherwise. The stable set polytope, denoted $\operatorname{STAB}(G)$, is the convex hull of the characteristic vectors of all stable sets in $G$, that is,

$$
\operatorname{STAB}(G):=\operatorname{conv}\left\{\chi^{S} \in \mathbb{R}^{V} \mid S \text { stable set of } G\right\} .
$$

Recall that a polytope $Q$ is an extension of a polytope $P$ if $P$ is the image of $Q$ under a linear projection.

LEMMA 8. For each $n$, there exists a graph $H_{n}$ with $O\left(n^{2}\right)$ vertices such that $\operatorname{STAB}\left(H_{n}\right)$ contains a face that is an extension of $\operatorname{COR}(n)$. 


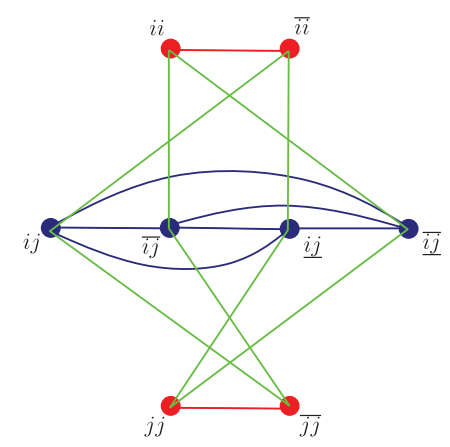

Fig. 1. The edges and vertices of $H_{n}$ corresponding to vertices $i, j$ and edge $i j$ of $K_{n}$.

Proof. Consider the complete graph $K_{n}$ with vertex set $V_{n}:=[n]$. For each vertex $i$ of $K_{n}$, we create two vertices labeled $i i, \overline{i i}$ in $H_{n}$ and an edge between them. Let us label the edges of $K_{n}$ in the following way. The edge between vertices $i$ and $j$ with $i<j$ gets the label $i j$. Now, for each edge $i j$ of $K_{n}$, we add to $H_{n}$ four vertices labeled $i j, \overline{i j}, \underline{i j}, \overline{i j}$ and all possible six edges between them. We further add the following eight edges to $H_{n}$ :

$$
\begin{aligned}
& \{i j, \overline{i i}\},\{i j, \overline{j j}\},\{\overline{i j}, i i\},\{\overline{i j}, \overline{j j}\}, \\
& \{\underline{i j}, \overline{i i}\},\{\underline{i j}, j j\},\{\underline{i j}, i i\},\{\underline{i j}, j j\} .
\end{aligned}
$$

See Figure 1 for an illustration. The number of vertices in $H_{n}$ is $2 n+4\left(\begin{array}{c}n \\ 2\end{array}\right)$.

Thus, the vertices and edges of $K_{n}$ are represented by cliques of size 2 and 4 respectively in $H_{n}$. We will refer to these as vertex-cliques and edge-cliques, respectively. Consider the face $F=F(n)$ of $\operatorname{STAB}\left(H_{n}\right)$ whose vertices correspond to the stable sets containing exactly one vertex in each vertex-clique and each edge-clique. (The vertices in this face correspond exactly to stable sets of $H_{n}$ with maximum cardinality.)

Consider the linear map $\pi: \mathbb{R}^{V\left(H_{n}\right)} \rightarrow \mathbb{R}^{n \times n}$ mapping a point $x \in \mathbb{R}^{V\left(H_{n}\right)}$ to the point $y \in \mathbb{R}^{n \times n}$ such that $y_{i j}=y_{j i}=x_{i j}$ for $i \leqslant j$. In this equation, the subscripts in $y_{i j}$ and $y_{j i}$ refer to an ordered pair of elements in [n], while the subscript in $x_{i j}$ refers to a vertex of $H_{n}$ that corresponds either to a vertex of $K_{n}$ (if $i=j$ ) or to an edge of $K_{n}$ (if $i \neq j$ ).

We claim that the image of $F$ under $\pi$ is $\operatorname{COR}(n)$, hence $F$ is an extension of $\operatorname{COR}(n)$; observe that it suffices to consider $0 / 1$ vertices as $F$ is a $0 / 1$ polytope and the projection is an orthogonal projection. Indeed, pick an arbitrary stable set $S$ of $H_{n}$ such that $x:=\chi^{S}$ is on face $F$. Then, define $b \in\{0,1\}^{n}$ by letting $b_{i}:=1$ if $i i \in S$ and $b_{i}:=0$ otherwise (i.e., $\overline{i i} \in S$ ). Notice that for the edge $i j$ of $K_{n}$ we have $i j \in S$ if and only if both vertices $i i$ and $j j$ belong to $S$. Hence, $\pi(x)=y=b b^{\top}$ is a vertex of $\operatorname{COR}(n)$. This proves $\pi(F) \subseteq \operatorname{COR}(n)$. Now pick a vertex $y:=b b^{\top}$ of $\operatorname{COR}(n)$ and consider the unique maximum stable set $S$ that contains vertex $i i$ if $b_{i}=1$ and vertex $\bar{i} i$ if $b_{i}=0$. Then, $x:=\chi^{S}$ is a vertex of $F$ with $\pi(x)=y$. Hence, $\pi(F) \supseteq \operatorname{COR}(n)$. Thus, $\pi(F)=\operatorname{COR}(n)$. This concludes the proof.

Our next lemma establishes simple monotonicity properties of the extension complexity used in our reduction.

Lemma 9. Let $P, Q$, and $F$ be polytopes. Then, the following hold:

(i) if $F$ is an extension of $P$, then $\mathrm{xc}(F) \geqslant \mathrm{xc}(P)$;

(ii) if $F$ is a face of $Q$, then $\operatorname{xc}(Q) \geqslant \operatorname{xc}(F)$. 
Proof. The first part is obvious because every extension of $F$ is in particular an extension of $P$. For the second part, notice that a slack matrix of $F$ can be obtained from the (facet-vs-vertex) slack matrix of $Q$ by deleting columns corresponding to vertices not in $F$. Now apply Theorem 3.

Using previous results, we can prove the following result about the worst-case extension complexity of the stable set polytope.

Theorem 10. For all $n$, one can construct a graph $G_{n}$ with $n$ vertices such that the extension complexity of the stable set polytope $\operatorname{STAB}\left(G_{n}\right)$ is $2^{\Omega(\sqrt{n})}$.

Proof. Without loss of generality, we may assume $n \geqslant 18$. For an integer $p \geqslant 3$, let $f(p):=\left|V\left(H_{p}\right)\right|=2 p+4\left(\begin{array}{l}p \\ 2\end{array}\right)$. Given $n \geqslant 18$, we define $p$ as the largest integer with $f(p) \leqslant n$. Now let $G_{n}$ be obtained from $H_{p}$ by adding $n-f(p)$ isolated vertices. Then $\operatorname{STAB}\left(H_{p}\right)$ is linearly isomorphic to a face of $\operatorname{STAB}\left(G_{n}\right)$. Using Theorem 7 in combination with Lemmas 8 and 9, we find that

$$
\begin{aligned}
\operatorname{xc}\left(\operatorname{STAB}\left(G_{n}\right)\right) & \geqslant \operatorname{xc}\left(\operatorname{STAB}\left(H_{p}\right)\right) \\
& \geqslant \operatorname{xc}(\operatorname{COR}(p)) \\
& =2^{\Omega(p)} \\
& =2^{\Omega(\sqrt{n})} .
\end{aligned}
$$

\subsection{TSP Polytopes}

Recall that $\operatorname{TSP}(n)$, the traveling salesman polytope or TSP polytope of $K_{n}=\left(V_{n}, E_{n}\right)$, is defined as the convex hull of the characteristic vectors of all subsets $F \subseteq E_{n}$ that define a tour of $K_{n}$. That is,

$$
\operatorname{TSP}(n):=\operatorname{conv}\left\{\chi^{F} \in \mathbb{R}^{E_{n}} \mid F \subseteq E_{n} \text { is a tour of } K_{n}\right\} .
$$

We now prove that the polytope $\operatorname{COR}(n)$ is the linear projection of a face of $\operatorname{TSP}\left(O\left(n^{2}\right)\right)$, implying the following.

LEMMA 11. For each $n$, there exists a positive integer $q=O\left(n^{2}\right)$ such that $\operatorname{TSP}(q)$ contains a face that is an extension of $\operatorname{COR}(n)$.

Proof. Recall that

$$
\operatorname{COR}(n)=\operatorname{conv}\left\{b b^{\top} \in \mathbb{R}^{n \times n} \mid b \in\{0,1\}^{n}\right\} .
$$

To prove the lemma, we start with constructing a graph $G_{n}$ with $q=O\left(n^{2}\right)$ vertices such that the tours of $G_{n}$ correspond to the $n \times n$ rank-1 binary symmetric matrices $b b^{\top}$, where $b \in\{0,1\}^{n}$. This is done in three steps:

(i) define a 3SAT formula $\phi_{n}$ with $n^{2}$ variables such that the satisfying assignments of $\phi_{n}$ bijectively correspond to the matrices $b b^{\top}$, where $b \in\{0,1\}^{n}$;

(ii) construct a directed graph $D_{n}$ with $O\left(n^{2}\right)$ vertices such that each directed tour of $D_{n}$ defines a satisfying assignment of $\phi_{n}$, and conversely each satisfying assignment of $\phi_{n}$ has at least one corresponding directed tour in $D_{n}$;

(iii) modify the directed graph $D_{n}$ into an undirected graph $G_{n}$ in such a way that the tours of $G_{n}$ bijectively correspond to the directed tours of $D_{n}$.

Step (i). For defining $\phi_{n}$ we use Boolean variables $C_{i j} \in\{0,1\}$ for $i, j \in[n]$ and let

$$
\phi_{n}:=\bigwedge_{\substack{i, j \in[n] \\ i \neq j}}\left[\left(C_{i i} \vee C_{j j} \vee \overline{C_{i j}}\right) \wedge\left(C_{i i} \vee \overline{C_{j j}} \vee \overline{C_{i j}}\right) \wedge\left(\overline{C_{i i}} \vee C_{j j} \vee \overline{C_{i j}}\right) \wedge\left(\overline{C_{i i}} \vee \overline{C_{j j}} \vee C_{i j}\right)\right]
$$




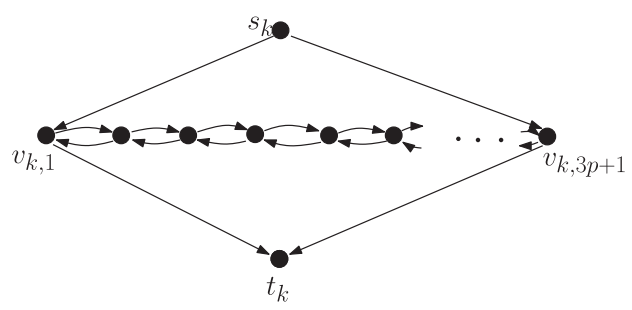

Fig. 2. Gadget for the $k$ th variable occurring in $p$ clauses.

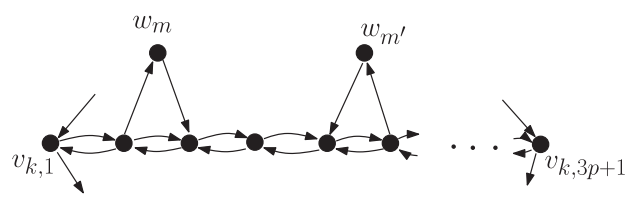

Fig. 3. Gadgets for clauses in case the $k$ th variable appears negated in the $m$ th clause and non-negated in the $m^{\prime}$ th clause.

The four clauses $\left(C_{i i} \vee C_{j j} \vee \overline{C_{i j}}\right),\left(C_{i i} \vee \overline{C_{j j}} \vee \overline{C_{i j}}\right),\left(\overline{C_{i i}} \vee C_{j j} \vee \overline{C_{i j}}\right)$, and $\left(\overline{C_{i i}} \vee \overline{C_{j j}} \vee C_{i j}\right)$ model the equation $C_{i j}=C_{i i} \wedge C_{j j}$. Hence, $C \in\{0,1\}^{n \times n}$ satisfies $\phi_{n}$ if and only if there exists $b \in\{0,1\}^{n}$ such that $C_{i j}=b_{i} \wedge b_{j}$ for all $i, j \in[n]$, or in matrix language, $C=b b^{\top}$.

Step (ii). To construct a directed graph $D_{n}$ whose directed tours correspond to the satisfying assignments of $\phi_{n}$ we use the standard reduction from 3SAT to HAMPATH [Sipser 1996].

We order the variables of $\phi_{n}$ arbitrarily and construct a gadget for each variable as follows. Suppose that the $k$ th variable occurs in $p$ clauses. We create a chain of $3 p+1$ nodes, labeled $v_{k, 1}, \ldots, v_{k, 3 p+1}$, where each node $v_{k, \ell}$ with $\ell<3 p+1$ is connected to the next node $v_{k, \ell+1}$ with two opposite directed edges. Figure 2 illustrates this. Traversing this chain from left to right is interpreted as setting the $k$ th variable to false and traversing it from right to left is interpreted as setting the $k$ th variable to true. We also have two nodes $s_{k}, t_{k}$ connected to this chain with directed edges $\left(s_{k}, v_{k, 1}\right),\left(s_{k}, v_{k, 3 p+1}\right)$, $\left(v_{k, 1}, t_{k}\right)$ and $\left(v_{k, 3 p+1}, t_{k}\right)$ creating a diamond structure.

Next, we order the clauses of $\phi_{n}$ arbitrarily and create a node for each clause. The node for the $m$ th clause is denoted by $w_{m}$. We connect these extra nodes to the gadgets for the variables as follows. Suppose, as before, that the $k$ th variable appears in $p$ clauses. Consider the $\ell$ th of these clauses in which the $k$ th variable appears, and let $m$ be the index of that clause. If the $k$ th variable appears negated in the $m$ th clause then we add the path $v_{k, 3 \ell-1}, w_{m}, v_{k, 3 \ell}$. Otherwise, the $k$ th variable appears nonnegated in the $m$ th clause and we add the path $v_{k, 3 \ell}, w_{m}, v_{k, 3 \ell-1}$. Figure 3 illustrates this.

Next we connect the gadgets corresponding to the variables by identifying $t_{k}$ with $s_{k+1}$ for $1 \leqslant k<n^{2}$. Finally, we add a directed edge from $t_{n^{2}}$ to $s_{1}$. Figure 4 illustrates the final directed graph obtained.

To see why the directed tours of the final directed graph $D_{n}$ define satisfying assignments of our Boolean formula $\phi_{n}$, observe that each directed tour of $D_{n}$ encodes a truth assignment to the $n^{2}$ variables depending on which way the corresponding chains are traversed. Because a directed tour visits every node and because the node $w_{m}$ corresponding to a clause can be visited only if we satisfy it, the truth assignment satisfies $\phi_{n}$. Conversely, every satisfying assignment of $\phi_{n}$ yields at least one directed tour in $D_{n}$. (If the $m$ th clause is satisfied by the value of more than one variable, we visit $w_{m}$ only once, from the chain of the first variable whose value makes the clause satisfied.) 


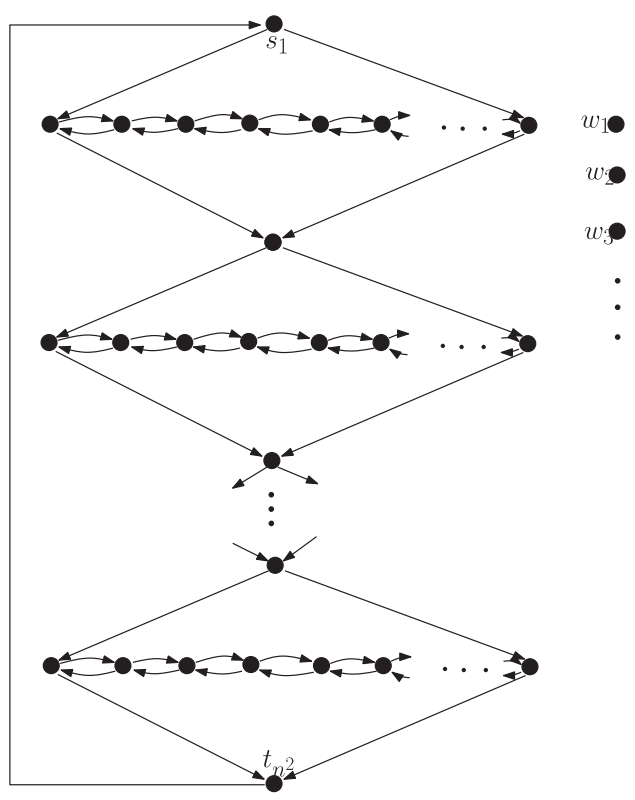

Fig. 4. Final graph. Directed edges incident to nodes $w_{m}$ depend on the actual ordering of variables and clauses in Boolean formula $\phi_{n}$.

Step (iii). For each node $v$ of $D_{n}$ we create a path $v_{\text {in }}, v_{\text {mid }}, v_{\text {out }}$ in the (undirected) graph $G_{n}$. For each directed edge $(v, w)$ of $D_{n}$, we add to the graph $G_{n}$ an edge between $v_{\text {out }}$ and $w_{\text {in }}$. As is easily seen, the tours of $G_{n}$ bijectively correspond to the directed tours of $D_{n}$. Note that $G_{n}$ has $q:=3\left(n(n-1) \cdot 13+n \cdot(3 n-2)+n^{2}+4 n(n-1)\right)=O\left(n^{2}\right)$ vertices.

Consider the face $F$ of $\operatorname{TSP}(q)$ defined by setting to 0 all variables $x_{e}$ corresponding to non-edges of $G_{n}$, so that the vertices of $F$ are the characteristic vectors of the tours of $G_{n}$. To conclude the proof, we give a linear projection $\pi: x \mapsto y:=\pi(x)$ mapping $F$ to $\operatorname{COR}(n)$. For $x \in \mathbb{R}^{E_{q}}$ and $i, j \in[n]$, we let $y_{i j}=x_{e}$, where $e$ is the edge $\left(v_{\text {out }, k, 2}, v_{i n, k, 1}\right)$ of $G_{n}$ corresponding to the directed edge $\left(v_{k, 2}, v_{k, 1}\right)$ and $k$ is the index of the variable $C_{i j}$ of $\phi_{n}$. It follows from this discussion that $\pi$ maps the face $F$ of $\operatorname{TSP}(q)$ to $\operatorname{COR}(n)$. The lemma follows.

The final theorem in this section follows from Theorem 7, Lemmas 9 and 11, using an argument similar to that used in the proof of Theorem 10.

THEOREM 12. The extension complexity of the TSP polytope $\operatorname{TSP}(n)$ is $2^{\Omega(\sqrt{n})}$.

\section{QUANTUM COMMUNICATION AND PSD FACTORIZATIONS}

In this section, we explain the connection with quantum communication. This yields results that are interesting in their own right, and also clarifies where the matrix $M$ of Section 2 came from.

For a general introduction to quantum computation we refer to Nielsen and Chuang [2000] and to Mermin [2007], and for quantum communication complexity we refer to de Wolf [2002] and to Buhrman et al. [2010]. For our purposes, an $r$-dimensional quantum state $\rho$ is an $r \times r$ PSD matrix of trace $1 .{ }^{7} \mathrm{~A} k$-qubit state is a state in dimension $r=2^{k}$. If $\rho$ has rank 1 , it can be written as an outer product $|\phi\rangle\langle\phi|$ of some unit column

\footnotetext{
${ }^{7}$ For simplicity, we restrict to real rather than complex entries, which does not significantly affect the results.
} 
vector $|\phi\rangle$ and its conjugate transpose $\langle\phi|$ (which is a row vector). This $|\phi\rangle$ is sometimes called a pure state. We use $|i\rangle$ to denote the pure state vector that has 1 at position $i$ and 0s elsewhere. A quantum measurement (POVM) is described by a set of PSD matrices $\left\{E_{\theta}\right\}_{\theta \in \Theta}$, each labeled by a real number $\theta$, and summing to the $r$-dimensional identity: $\sum_{\theta \in \Theta} E_{\theta}=I$. When measuring state $\rho$ with this measurement, the probability of outcome $\theta$ equals $\operatorname{Tr}\left[E_{\theta} \rho\right]$. Note that if we define the PSD matrix $E:=\sum_{\theta \in \Theta} \theta E_{\theta}$, then the expected value of the measurement outcome is $\sum_{\theta \in \Theta} \theta \operatorname{Tr}\left[E_{\theta} \rho\right]=\operatorname{Tr}\left[E_{\rho}\right]$.

\subsection{PSD Factorizations}

Analogous to nonnegative factorizations and nonnegative rank, one can define PSD factorizations and PSD rank. A rank-r PSD factorization of an $m \times n$ matrix $M$ is a collection of $r \times r$ symmetric positive semidefinite matrices $T_{1}, \ldots, T_{m}$ and $U^{1}, \ldots, U^{n}$ such that the Frobenius product $\left\langle T_{i}, U^{j}\right\rangle=\operatorname{Tr}\left[\left(T_{i}\right)^{\top} U^{j}\right]=\operatorname{Tr}\left[T_{i} U^{j}\right]$ equals $M_{i j}$ for all $i \in[m], j \in[n]$. The PSD rank of $M$ is the minimum $r$ such that $M$ has a rank-r PSD factorization. We denote this by $\operatorname{rank}_{\mathrm{PSD}}(M)$.

Here, we show that $\operatorname{rank}_{\mathrm{PSD}}(M)$ can be expressed in terms of the amount of communication needed by a one-way quantum communication protocol for computing $M$ in expectation (Corollary 15). Before doing so, we state the geometric interpretation of $\operatorname{rank}_{\mathrm{PSD}}(M)$ when $M$ is a slack matrix.

For a positive integer $r$, we let $\mathbb{S}_{+}^{r}$ denote the cone of $r \times r$ symmetric positive semidefinite matrices embedded in $\mathbb{R}^{r(r+1) / 2}$ in such a way that, for all $y, z \in \mathbb{S}_{+}^{r}$, the scalar product $z^{\top} y$ is the Frobenius product of the corresponding matrices. A semidefinite $E F$ of size $r$ is a conic EF with respect to $C=\mathbb{S}_{+}^{r}$, that is, a system $E f+F y=g$, $y \in \mathbb{S}_{+}^{r}$ such that $P=\left\{x \in \mathbb{R}^{d} \mid \exists y: E f+F y=g, y \in \mathbb{S}_{+}^{r}\right\}$. We call the set $Q=\left\{(x, y) \in \mathbb{R}^{d+r(r+1) / 2} \mid E x+F y=g, y \in \mathbb{S}_{+}^{r}\right\}$ a semidefinite extension of $P$. The semidefinite extension complexity of polytope $P$, denoted by $\operatorname{xc}_{\mathrm{SDP}}(P)$, is the minimum $r$ such that $P$ has a semidefinite EF of size $r$. Observe that $\left(\mathbb{S}_{+}^{r}\right)^{*}=\mathbb{S}_{+}^{r}$.

The following result follows from Gouveia et al. [2013]:

Theorem 13. Let $P=\left\{x \in \mathbb{R}^{d} \mid A x \leqslant b\right\}=\operatorname{conv}(V)$ be a polytope of dimension at least 1 . Then the slack matrix $S$ of $P$ with respect to $A x \leqslant b$ and $V$ has a factorization $S=T U$ so that $\left(T_{i}\right)^{\top}, U^{j} \in \mathbb{S}_{+}^{r}$ if and only if there exists a semidefinite extension $Q=\left\{(x, y) \in \mathbb{R}^{d+r(r+1) / 2} \mid E x+F y=g, y \in \mathbb{S}_{+}^{r}\right\}$ with $P=\pi_{x}(Q)$.

\subsection{Quantum Protocols}

A one-way quantum protocol with r-dimensional messages can be described as follows. On input $i$, Alice sends Bob an $r$-dimensional state $\rho_{i}$. On input $j$, Bob measures the state he receives with a $\operatorname{POVM}\left\{E_{\theta}^{j}\right\}$ for some nonnegative values $\theta$, and outputs the result. We say that such a protocol computes a matrix $M$ in expectation, if the expected value of the output on respective inputs $i$ and $j$, equals the matrix entry $M_{i j}$. Analogous to the equivalence between classical protocols and nonnegative factorizations of $M$ established by Faenza et al. [2011], such quantum protocols are essentially equivalent to PSD factorizations of $S$.

TheOREM 14. Let $M \in \mathbb{R}_{+}^{m \times n}$ be a matrix. Then the following holds.

(i) A one-way quantum protocol with $r$-dimensional messages that computes $M$ in expectation, gives a rank-r PSD factorization of $M$.

(ii) A rank-r PSD factorization of $M$ gives a one-way quantum protocol with $(r+1)$ dimensional messages that computes $M$ in expectation. 
Proof. The first part is straightforward. Given a quantum protocol as above, define $E^{j}:=\sum_{\theta \in \Theta} \theta E_{\theta}^{j}$. Clearly, on inputs $i$ and $j$ the expected value of the output is $\operatorname{Tr}\left[\rho_{i} E^{j}\right]=$ $M_{i j}$.

For the second part, suppose we are given a PSD factorization of a matrix $M$, so we are given PSD matrices $T_{1}, \ldots, T_{m}$ and $U^{1}, \ldots, U^{n}$ satisfying $\operatorname{Tr}\left[T_{i} U^{j}\right]=M_{i j}$ for all $i, j$. In order to turn this into a quantum protocol, define $\tau=\max _{i} \operatorname{Tr}\left[T_{i}\right]$. Let $\rho_{i}$ be the $(r+1)$-dimensional quantum state obtained by adding a $(r+1)$ st row and column to $T_{i} / \tau$, with $1-\operatorname{Tr}\left[T_{i}\right] / \tau$ as $(r+1)$ st diagonal entry, and 0 s elsewhere. Note that $\rho_{i}$ is indeed a PSD matrix of trace 1 , so it is a well-defined quantum state. For input $j$, derive Bob's $(r+1)$-dimensional POVM from the PSD matrix $U^{j}$ as follows. Let $\lambda$ be the largest eigenvalue of $U^{j}$, and define $E_{\tau \lambda}^{j}$ to be $U^{j} / \lambda$, extended with a $(r+1)$ st row and column of $0 \mathrm{~s}$. Let $E_{0}^{j}=I-E_{\tau \lambda}^{j}$. This is positive semidefinite because the largest eigenvalue of $E_{\tau \lambda}^{j}$ is 1 . Hence, the two operators $E_{\tau \lambda}^{j}$ and $E_{0}^{j}$ together form a well-defined POVM. The expected outcome (on inputs $i, j$ ) of the protocol induced by the states and POVMs that we just defined, is

$$
\tau \lambda \operatorname{Tr}\left[E_{\tau \lambda}^{j} \rho_{i}\right]=\operatorname{Tr}\left[T_{i} U^{j}\right]=M_{i j}
$$

so the protocol indeed computes $M$ in expectation.

We obtain the following corollary which summarizes the characterization of semidefinite EFs:

Corollary 15. For a polytope $P$ with slack matrix $S$, the following are equivalent:

(i) $P$ has a semidefinite extension $Q=\left\{(x, y) \in \mathbb{R}^{d+r(r+1) / 2} \mid E x+F y=g, y \in \mathbb{S}_{+}^{r}\right\}$;

(ii) the slack matrix $S$ has a rank-r PSD factorization;

(iii) there exists a one-way quantum communication protocol with $(r+1)$-dimensional messages (i.e., using $\lceil\log (r+1)\rceil$ qubits) that computes $S$ in expectation (for the converse we consider r-dimensional messages).

\subsection{A General Upper Bound on Quantum Communication}

Now we provide a quantum protocol that efficiently computes a nonnegative matrix $M$ in expectation, whenever there is a low rank matrix $N$ whose entry-wise square is $M$.

THEOREM 16. Let $M$ be a matrix with nonnegative real entries, $N$ be a rank-r matrix of the same dimensions such that $M_{i j}=N_{i j}^{2}$. Then there exists a one-way quantum protocol using $(r+1)$-dimensional pure-state messages that computes $M$ in expectation.

PRoOF. By Corollary 15, it suffices to give a rank-r PSD factorization of $M$. To this end, let $t_{i}, u_{j}$ be $r$-dimensional real vectors such that $N_{i j}=t_{i}^{\top} u_{j}$; such vectors exist because $N$ has rank $r$. Define $r \times r$ PSD matrices $T_{i}:=t_{i} t_{i}^{\top}$ and $U^{j}:=u_{j} u_{j}^{\top}$. Then

$$
\operatorname{Tr}\left[T_{i} U^{j}\right]=\left(t_{i}^{\top} u_{j}\right)^{2}=N_{i j}^{2}=M_{i j}
$$

hence we have a rank-r PSD factorization of $M$.

Note that, if $M$ is a $0 / 1$-matrix, then we may take $N=M$, hence any low-rank $0 / 1$ matrix can be computed in expectation by an efficient quantum protocol. If this $M$ is the slack matrix for a polytope $P \subseteq \mathbb{R}^{d}$, then it is easy to see that its rank is at most $d+1$ : the slack $M_{i j}=b_{i}-A_{i} v_{j}$ of a constraint $A_{i} x \leqslant b_{i}$ with respect to a point $v_{j} \in P$ can be written as the inner product between the two $(d+1)$-dimensional vectors $\left(b_{i},-A_{i}\right)$ and $\left(1, v_{j}\right)$. We thus obtain the following corollary (implicit in Theorem 4.2 of Gouveia et al. [2010]) which also implies a compact (i.e., polynomial size) semidefinite EF for the stable set polytope of perfect graphs, reproving the previously known result by 
Lovász [1979, 2003]. We point out that the result still holds when $\operatorname{dim}(P)+2$ is replaced by $\operatorname{dim}(P)+1$, see Gouveia et al. [2013]; this difference is due to normalization.

Corollary 17. Let $P$ be a polytope such that $S(P)$ is a $0 / 1$ matrix. Then $\operatorname{xc}_{\mathrm{SDP}}(P) \leqslant$ $\operatorname{dim}(P)+2$.

\subsection{Quantum vs Classical Communication, and PSD vs Nonnegative Factorizations}

We now give an example of an exponential separation between quantum and classical communication in expectation, based on the matrix $M$ of Section 2 . This result actually preceded and inspired the results in Section 3.

THEOREM 18. For each $n$, there exists a nonnegative matrix $M \in \mathbb{R}^{2^{n} \times 2^{n}}$ that can be computed in expectation by a quantum protocol using $\log n+O(1)$ qubits, while any classical randomized protocol needs $\Omega(n)$ bits to compute $M$ in expectation.

Proof. Consider the matrix $N \in \mathbb{R}^{2^{n} \times 2^{n}}$ whose rows and columns are indexed by $n$-bit strings $a$ and $b$, respectively, and whose entries are defined as $N_{a b}=1-a^{\top} b$. Define $M \in \mathbb{R}_{+}^{2^{n} \times 2^{n}}$ by $M_{a b}=N_{a b}^{2}$. This $M$ is the matrix from Section 2 . Note that $N$ has rank $r \leqslant n+1$ because it can be written as the sum of $n+1$ rank-1 matrices. Hence, Theorem 16 immediately implies a quantum protocol with $(n+2)$-dimensional messages that computes $M$ in expectation.

For the classical lower bound, note that a protocol that computes $M$ in expectation has positive probability of giving a nonzero output on input $a, b$ if and only if $M_{a b}>0$. With a message $m$ in this protocol we can associate a rectangle $R_{m}=A \times B$ where $A$ consists of all inputs $a$ for which Alice has positive probability of sending $m$, and $B$ consists of all inputs $b$ for which Bob, when he receives message $m$, has positive probability of giving a nonzero output. Together these rectangles will cover exactly the nonzero entries of $M$. Accordingly, a $c$-bit protocol that computes $M$ in expectation induces a rectangle cover for the support matrix of $M$ of size $2^{c}$. Theorem 1 lower bounds the size of such a cover by $2^{\Omega(n)}$, hence $c=\Omega(n)$.

Together with Theorem 14 and the equivalence of randomized communication complexity (in expectation) and nonnegative rank established in Faenza et al. [2011], we immediately obtain an exponential separation between nonnegative rank and PSD rank.

Corollary 19. For each $n$, there exists $M \in \mathbb{R}_{+}^{2^{n} \times 2^{n}}$, with $\operatorname{rank}_{+}(M)=2^{\Omega(n)}$ and $\operatorname{rank}_{\mathrm{PSD}}(M)=O(n)$.

In fact a simple rank-( $n+1)$ PSD factorization of $M$ is the following: let $T_{a}:=\left(\begin{array}{c}1 \\ -a\end{array}\right)\left(\begin{array}{c}1 \\ -a\end{array}\right)^{\top}$ and $U^{b}:=\left(\begin{array}{l}1 \\ b\end{array}\right)\left(\begin{array}{l}1 \\ b\end{array}\right)^{\top}$, then $\operatorname{Tr}\left[T_{a} U^{b}\right]=\left(1-a^{\top} b\right)^{2}=M_{a b}$.

\section{CONCLUDING REMARKS}

In addition to proving the first unconditional super-polynomial lower bounds on the size of linear EFs for the cut polytope, stable set polytope, and TSP polytope, we demonstrate that the rectangle covering bound can prove strong results in the context of EFs. In particular, it can be super-polynomial in the dimension and the logarithm of the number of vertices of the polytope, settling an open problem of Fiorini et al. [2011].

The exponential separation between nonnegative rank and PSD rank that we prove here (Theorem 18) actually implies more than a super-polynomial lower bound on the extension complexity of the cut polytope. As noted in Theorem 5 , the polytopes CUT $(n)$ and $\operatorname{COR}(n-1)$ are affinely isomorphic. Let $Q(n)$ denote the polyhedron isomorphic (under the same affine map) to the polyhedron defined by (5) for $a \in\{0,1\}^{n}$. Then (i) every polytope (or polyhedron) that contains $\operatorname{CUT}(n)$ and is contained in $Q(n)$ has 
exponential extension complexity; (ii) there exists a low complexity spectrahedron that contains $\operatorname{CUT}(n)$ and is contained in $Q(n)$. (A spectrahedron is any projection of an affine slice of the positive semidefinite cone.) This was used in Braun et al. [2012] to establish the existence of a spectrahedron that cannot be well approximated by linear programs of polynomial size.

An important problem also left open in Yannakakis [1991] is whether the perfect matching polytope has a polynomial-size linear EF. Yannakakis proved that every symmetric $\mathrm{EF}$ of this polytope has exponential size, a striking result given the fact that the perfect matching problem is solvable in polynomial time. He conjectured that asymmetry also does not help in the case of the perfect matching polytope. Because it is based on the rectangle covering bound, our argument does not yield a super-polynomial lower bound on the extension complexity of the perfect matching polytope. This question was recently answered in the affirmative in Rothvoss [2014], showing that the extension complexity of the perfect matching polytope is $2^{\Omega(n)}$. This groundbreaking result is based on a general lower bound called the hyperplane separation bound, which was used implicitly, for example, in Braun et al. [2012].

As mentioned at the end of the introduction, the new connections developed have already inspired much follow-up research in particular about approximate EFs. Here are two concrete questions left open for future work: (i) find a slack matrix that has an exponential gap between nonnegative rank and PSD rank; (ii) prove that the cut polytope has no polynomial-size semidefinite EF (that would rule out SDP-based algorithms for optimizing over the cut polytope, in the same way that this article ruled out LP-based algorithms).

Our final remark concerns the famous log-rank conjecture [Lovász and Saks 1993]. It states that the deterministic communication complexity of a (finite) Boolean matrix $M$ is upper bounded by a polynomial in the logarithm of its $\operatorname{rank} \operatorname{rank}(M)$. On the one hand, this conjecture is equivalent to the following statement: $\log \left(\operatorname{rank}_{+}(M)\right) \leqslant$ polylog$(\operatorname{rank}(M))$ for all Boolean matrices $M$. On the other hand, we know that $\operatorname{rank}_{\mathrm{PSD}}(M)=O(\operatorname{rank}(M))$ for all Boolean matrices $M$ by Theorem 16 . Using the interpretation of the nonnegative and PSD rank of $M$ in terms of classical and quantum communication protocols computing $M$ in expectation (see Faenza et al. [2011] and Theorem 14), we see that the log-rank conjecture is equivalent to the conjecture that classical protocols computing $M$ in expectation are at most polynomially less efficient than quantum protocols. Accordingly, one way to prove the log-rank conjecture would be to give an efficient classical simulation of such quantum protocols for Boolean $M$ (for non-Boolean $M$, we already exhibited an exponential separation in this article).

\section{APPENDIX}

\section{A. BACKGROUND ON POLYTOPES}

A (convex) polytope is a set $P \subseteq \mathbb{R}^{d}$ that is the convex hull conv $(V)$ of a finite set of points $V$. Equivalently, $P$ is a polytope if and only if $P$ is bounded and the intersection of a finite collection of closed halfspaces. This is equivalent to saying that $P$ is bounded and the set of solutions of a finite system of linear inequalities and possibly equalities (each of which can be represented by a pair of inequalities).

Let $P \subseteq \mathbb{R}^{d}$ be a polytope. A closed halfspace $H^{+}$that contains $P$ is said to be valid for $P$. In this case, the hyperplane $H$ that bounds $H^{+}$is also said to be valid for $P$. A face of $P$ is either $P$ itself or the intersection of $P$ with a valid hyperplane. Every face of a polytope is again a polytope. A face is called proper if it is not the polytope itself. A vertex is a minimal nonempty face. A facet is a maximal proper face. An inequality $c^{\top} x \leqslant \delta$ is said to be valid for $P$ if it is satisfied by all points of $P$. The face it defines 
is $F:=\left\{x \in P \mid c^{\top} x=\delta\right\}$. The inequality is called facet-defining if $F$ is a facet. The dimension of a polytope $P$ is the dimension of its affine hull aff $(P)$.

Every (finite or infinite) set $V$ such that $P=\operatorname{conv}(V)$ contains all the vertices of $P$. Conversely, letting vert $(P)$ denote the vertex set of $P$, we have $P=\operatorname{conv}(\operatorname{vert}(P))$. Suppose now that $P$ is full dimensional, that is, $\operatorname{dim}(P)=d$. Then, every (finite) system $A x \leqslant b$ such that $P=\left\{x \in \mathbb{R}^{d} \mid A x \leqslant b\right\}$ contains all the facet-defining inequalities of $P$, up to scaling by positive numbers. Conversely, $P$ is described by its facet-defining inequalities.

If $P$ is not full dimensional, these statements have to be adapted as follows. Every (finite) system describing $P$ contains all the facet-defining inequalities of $P$, up to scaling by positive numbers and adding an inequality that is satisfied with equality by all points of $P$. Conversely, a linear description of $P$ can be obtained by picking one inequality per facet and adding a system of equalities describing aff $(P)$.

A 0/1-polytope in $\mathbb{R}^{d}$ is simply the convex hull of a subset of $\{0,1\}^{d}$.

A (convex) polyhedron is a set $P \subseteq \mathbb{R}^{d}$ that is the intersection of a finite collection of closed halfspaces. A polyhedron $P$ is a polytope if and only if it is bounded.

For more background on polytopes and polyhedra, see the standard reference [Ziegler 1995].

Note added in proof. Lee et al. [2015] have very recently proved super-polynomial lower bounds on the semidefinite extension complexity of the cut, TSP, and stable set polytopes, thereby answering one of the open questions raised in Section 5.

\section{ACKNOWLEDGMENTS}

We thank Kota Ishihara for carefully reading the manuscript and pointing out an error in a previous version of the text. We thank Monique Laurent for information about hypermetric inequalities, and the three anonymous STOC'12 referees as well as one JACM referee for suggesting improvements to the text. Sebastian Pokutta would like to thank Alexander Martin for the inspiring discussions and support. Ronald de Wolf thanks Giannicola Scarpa and Troy Lee for useful discussions.

\section{REFERENCES}

S. Aaronson. 2006. Lower bounds for local search by quantum arguments. SIAM J. Comput. 35, 4, 804-824. (Earlier version in STOC'04).

D. Aharonov and O. Regev. 2004. Lattice problems in NP $\cap$ coNP. In Proceedings of FOCS 2004. $362-371$.

S. Arora, B. Bollobás, and L. Lovász. 2002. Proving integrality gaps without knowing the linear program. In Proceedings of FOCS 2002. 313-322.

S. Arora, B. Bollobás, L. Lovász, and I. Tourlakis. 2006. Proving integrality gaps without knowing the linear program. Theory Comput. 2, 19-51.

D. Avis and H. R. Tiwary. 2013. On the Extension Complexity of Combinatorial Polytopes. In Proceedings of ICALP(1) 2013. 57-68.

E. Balas. 1985. Disjunctive programming and a hierarchy of relaxations for discrete optimization problems. SIAM J. Algeb. Disc. Meth. 6, 466-486.

E. Balas, S. Ceria, and G. Cornuéjols. 1993. A lift-and-project algorithm for mixed 0-1 programs. Math. Prog. 58, 295-324.

S. Benabbas and A. Magen. 2010. Extending SDP integrality gaps to Sherali-Adams with applications to quadratic programming and MaxCutGain. In Proceedings of IPCO 2010. 299-312.

G. Braun, S. Fiorini, S. Pokutta, and D. Steurer. 2012. Approximation limits of linear programs (beyond hierarchies). In Proceedings of FOCS 2012. 480-489.

G. Braun, S. Fiorini, and S. Pokutta. 2013a. Average case polyhedral complexity of the maximum stable set problem. arXiv:1311.4001.

G. Braun, R. Jain, T. Lee, and S. Pokutta. 2013b. Information-theoretic approximations of the nonnegative rank. ECCC Report no. 158 (2013).

G. Braun and S. Pokutta. 2013. Common information and unique disjointness. In Proceedings of FOCS 2013. 688-697. 
M. Braverman and A. Moitra. 2013. An information complexity approach to extended formulations. In Proceedings of STOC 2013. 161-170.

J. Briët, D. Dadush, and S. Pokutta. 2013. On the existence of 0/1 polytopes with high semidefinite extension complexity. In Algorithms ESA 2013, Lecture Notes in Computer Science, vol. 8125, Springer, 217-228.

H. Buhrman, R. Cleve, S. Massar, and R. de Wolf. 2010. Nonlocality and communication complexity. Rev. Modern Phys. 82, 665.

J. Buresh-Oppenheim, N. Galesi, S. Hoory, A. Magen, and T. Pitassi. 2006. Rank bounds and integrality gaps for cutting planes procedures. Theory Comput. 2, 65-90.

S. O. Chan, J. R. Lee, P. Raghavendra, and D. Steurer. 2013. Approximate Constraint Satisfaction Requires Large LP Relaxations. In Proceedings of FOCS 2013, 350-359.

M. Charikar, K. Makarychev, and Y. Makarychev. 2009. Integrality gaps for Sherali-Adams relaxations. In Proceedings of STOC 2009. 283-292.

M. Conforti, G. Cornuéjols, and G. Zambelli. 2010. Extended formulations in combinatorial optimization. $4 O R$ 8, 1-48.

G. B. Dantzig. 1951. Maximization of a linear function of variables subject to linear inequalities. In Activity Analysis of Production and Allocation, Cowles Commission Monograph No. 13, John Wiley \& Sons Inc., New York, 339-347.

C. De Simone. 1990. The cut polytope and the Boolean quadric polytope. Disc. Math. 79, 71-75.

M. M. Deza and M. Laurent. 1997. Geometry of Cuts and Metrics. Algorithms and Combinatorics Series, vol. 15, Springer-Verlag.

A. Drucker and R. de Wolf. 2011. Quantum proofs for classical theorems. Theory Comput. Graduate Surveys 2.

Y. Faenza, S. Fiorini, R. Grappe, and H. R. Tiwary. 2011. Extended formulations, non-negative factorizations and randomized communication protocols. arXiv:1105.4127.

H. Fawzi and P. A. Parrilo. 2013. Exponential lower bounds on fixed-size PSD rank and semidefinite extension complexity. arXiv:1311.2571.

W. Fernandez de la Vega and C. Mathieu. 2007. Linear programming relaxation of Maxcut. In Proceedings of SODA 2007. 53-61.

S. Fiorini, V. Kaibel, K. Pashkovich, and D. O. Theis. 2011. Combinatorial bounds on nonnegative rank and extended formulations. arXiv:1111.0444.

S. Fiorini, S. Massar, M. K. Patra, and H. R. Tiwary. 2013. Generalised probabilistic theories and conic extensions of polytopes. CoRR abs/1310.4125.

K. Georgiou, A. Magen, T. Pitassi, and I. Tourlakis. 2010. Integrality gaps of $2-o(1)$ for vertex cover SDPs in the Lovász-Schrijver hierarchy. SIAM J. Comput. 39, 3553-3570.

K. Georgiou, A. Magen, and M. Tulsiani. 2009. Optimal Sherali-Adams gaps from pairwise independence. In Proceedings of APPROX-RANDOM 2009. 125-139.

M. X. Goemans and D. P. Williamson. 1995. Improved approximation algorithms for maximum cut and satisfiability problems using semidefinite programming. J. ACM 42, 1115-1145.

J. Gouveia, P. A. Parrilo, and R. R. Thomas. 2010. Theta bodies for polynomial ideals. SIAM J. Optim. 20, 2097-2118.

J. Gouveia, P. A. Parrilo, and R. R. Thomas. 2013. Lifts of convex sets and cone factorizations. Math. Oper. Res. 38, 2, 248-264.

J. Håstad. 1999. Clique is Hard to Approximate within $n^{1-\epsilon}$. Acta Math. 182, 105-142. (Earlier version in Proceedings of FOCS 1996.)

H. Huang and B. Sudakov. 2012. A counterexample to the Alon-Saks-Seymour conjecture and related problems. Combinatorica 32, 2, 205-219.

R. Jain, Y. Shi, Z. Wei, and S. Zhang. 2013. Efficient protocols for generating bipartite classical distributions and quantum states. IEEE Trans. Inf. Theory 59, 8, 5171-5178.

V. Kaibel. 2011. Extended formulations in combinatorial optimization. Optima 85, 2-7.

V. Kaibel, K. Pashkovich, and D. O. Theis. 2010. Symmetry matters for the sizes of extended formulations. In Proceedings of IPCO 2010. 135-148.

V. Kaibel and S. Weltge. 2013. A short proof that the extension complexity of the correlation polytope grows exponentially. Discrete Computa. Geom. 53, 2, 396-401.

N. Karmarkar. 1984. A new polynomial time algorithm for linear programming. Combinatorica 4, 373-395.

I. Kerenidis and R. de Wolf. 2004. Exponential lower bound for 2-query locally decodable codes via a quantum argument. J. Comput. Syst. Sci. 69, 3, 395-420. (Earlier version in STOC 2003). 
L. G. Khachiyan. 1979. A polynomial algorithm in linear programming. Dokl. Akad. Nauk SSSR 244, 5, 1093-1096.

S. Khot, G. Kindler, E. Mossel, and R. O'Donnell. 2007. Optimal inapproximability results for MAX-CUT and other 2-variable CSPs? SIAM J. Comput. 37, 1, 319-357.

H. Klauck, T. Lee, and S. Zhang. 2011. An explicit and exponential separation between randomized and quantum correlation complexities. Unpublished manuscript from Oct/Nov 2011. Personal communication between Troy Lee and Ronald de Wolf, December 2011 at Centre for Quantum Technologies, Singapore.

E. Kushilevitz and N. Nisan. 1997. Communication Complexity. Cambridge University Press.

E. Kushilevitz and E. Weinreb. 2009a. The communication complexity of set-disjointness with small sets and 0-1 intersection. In Proceedings of FOCS 2009. 63-72.

E. Kushilevitz and E. Weinreb. 2009b. On the complexity of communication complexity. In Proceedings of STOC 2009. 465-474.

J. R. Lee, P. Raghavendra, and D. Steurer. 2015. Lower bounds on the size of semidefinite programming relaxations. In Proceedings of STOC 2015.

T. Lee and D. O. Theis. 2012. Support-based lower bounds for the positive semidefinite rank of a nonnegative matrix. arXiv:1203.3961.

L. Lovász. 1979. On the Shannon capacity of a graph. IEEE Trans. Inform. Theory 25, 1-7.

L. Lovász. 2003. Semidefinite programs and combinatorial optimization. In Recent Advances in Algorithms and Combinatorics. CMS Books Math./Ouvrages Math, SMC Series, vol. 11, Springer, 137-194.

L. Lovász and M. Saks. 1993. Communication complexity and combinatorial lattice theory. J. Comput. Syst. Sci. $47,322-349$.

L. Lovász and A. Schrijver. 1991. Cones of matrices and set-functions and 0-1 optimization. SIAM J. Optim. $1,166-190$.

N. D. Mermin. 2007. Quantum Computer Science: An Introduction. Cambridge University Press.

M. A. Nielsen and I. L. Chuang. 2000. Quantum Computation and Quantum Information. Cambridge University Press.

K. Pashkovich. 2009. Symmetry in extended formulations of the permutahedron. arXiv:0912.3446.

S. Pokutta and M. Van Vyve. 2013. A note on the extension complexity of the knapsack polytope. Oper. Res. Lett. 41, 4, 347-350.

A. A. Razborov. 1992. On the distributional complexity of disjointness. Theoret. Comput. Sci. 106, 2, 385-390.

T. Rothvoss. 2011. Some 0/1 polytopes need exponential size extended formulations. arXiv:1105.0036.

T. Rothvoss. 2014. The matching polytope has exponential extension complexity. In Proceedings of STOC 2014. 263-272.

G. Schoenebeck, L. Trevisan, and M. Tulsiani. 2007. Tight integrality gaps for Lovasz-Schrijver LP relaxations of vertex cover and max cut. In Proceedings of STOC 2007. 302-310.

A. Schrijver. 2003. Combinatorial Optimization. Polyhedra and Efficiency. Springer-Verlag.

C. E. Shannon. 1949. The synthesis of two-terminal switching circuits. Bell Syst. Tech. J. 25, 59-98.

H. D. Sherali and W. P. Adams. 1990. A hierarchy of relaxations between the continuous and convex hull representations for zero-one programming problems. SIAM J. Disc. Math. 3, 411-430.

M. Sipser. 1996. Introduction to the Theory of Computation. PWS, Boston, MA.

E. R. Swart. 1986; revision 1987. P = NP. Tech. rep., University of Guelph.

F. Vanderbeck and L. A. Wolsey. 2010. Reformulation and decomposition of integer programs. In 50 Years of Integer Programming 1958-2008, M. Jünger, Th, M. Liebling, D. Naddef, G. L. Nemhauser, W. R. Pulleyblank, G. Reinelt, G. Rinaldi, and L. A. Wolsey, Eds., Springer, 431-502.

R. de Wolf. 2002. Quantum communication and complexity. Theoret. Comput. Sci. 287, 337-353.

R. de Wolf. 2003. Nondeterministic quantum query and communication complexities. SIAM J. Comput. 32, 681-699.

L. A. Wolsey. 2011. Using extended formulations in practice. Optima 85, 7-9.

M. Yannakakis. 1988. Expressing combinatorial optimization problems by linear programs (extended abstract). In Proceedings of STOC 1988. 223-228.

M. Yannakakis. 1991. Expressing combinatorial optimization problems by linear programs. J. Comput. System Sci. 43, 3, 441-466.

G. M. Ziegler. 1995. Lectures on Polytopes. Graduate Texts in Mathematics, vol. 152. Springer-Verlag.

Received July 2012; revised November 2013 and September 2014; accepted January 2015 